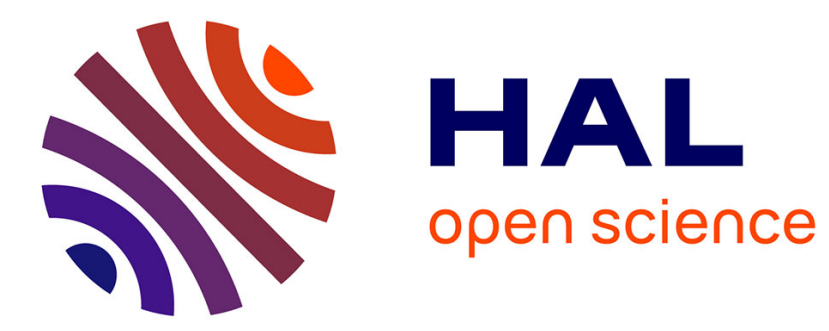

\title{
Les instruments juridiques français et européens dans la mise en place du principe d'égalité et de non-discrimination
}

Daniel Borrillo

\section{- To cite this version:}

Daniel Borrillo. Les instruments juridiques français et européens dans la mise en place du principe d'égalité et de non-discrimination. Revue française des affaires sociales, 2002, Protection sociale aux Etats-Unis: évaluations, réformes et débats, 56 (1 Janvier-Mars). hal-01238697

\section{HAL Id: hal-01238697 \\ https://hal.science/hal-01238697}

Submitted on 6 Dec 2015

HAL is a multi-disciplinary open access archive for the deposit and dissemination of scientific research documents, whether they are published or not. The documents may come from teaching and research institutions in France or abroad, or from public or private research centers.
L'archive ouverte pluridisciplinaire HAL, est destinée au dépôt et à la diffusion de documents scientifiques de niveau recherche, publiés ou non, émanant des établissements d'enseignement et de recherche français ou étrangers, des laboratoires publics ou privés. 


\section{Les instruments juridiques français et européens dans la mise en place du principe d'égalité et de non-discrimination ${ }^{1}$}

Daniel Borrillo*

Dans un univers aux ressources (matérielles et/ou symboliques) limitées, la question de l'accès à ces dernières constitue l'un des enjeux politiques majeurs des États démocratiques. Depuis fort longtemps, les théoriciens de la politique ont réfléchi aux différents systèmes permettant la répartition des biens (économiques, culturels, sociaux, spirituels...) au sein de la communauté. Les premiers critères de distribution entre les individus trouvaient leur légitimation dans l'appartenance à une lignée ou à un groupe social spécifique. Ainsi, la naissance au sein d'une famille justifiait-elle l'octroi de certains privilèges. En Europe, ce n'est que récemment que ce critère politique a été contesté radicalement. En effet, il faudra attendre la fin du XVIII ${ }^{\mathrm{e}}$ siècle pour que la Révolution française mette fin au système de castes en le dénonçant comme contraire aux droits fondamentaux de l'individu. Considérée comme un privilège arbitraire et par conséquent essentiellement immoral, dans l'idéal républicain, la naissance ne constituera plus un élément légitimant la concession de biens économiques, culturels et politiques : dorénavant le mérite sera l'attribut exclusif de classement des individus ${ }^{2}$. La Déclaration des droits de l'homme et du citoyen de 1789 place ceux-ci au centre du système politique : l'État est conçu en fonction de l'individu et pour lui garantir la jouissance de ses droits « naturels ». La Déclaration proclame la liberté et l'égalité de tous: "les hommes naissent et demeurent libres et égaux en droit », et l'État doit se porter garant de cette égalité : «la loi doit être la même pour tous, soit qu'elle protège, soit qu'elle punisse ». Par la suite, l'ensemble des chartes, des déclarations et des conventions internationales relatives aux droits humains confirmeront la prééminence du postulat d'égalité devant la loi comme constitutif du régime démocratique ${ }^{3}$.

\footnotetext{
* Juriste, université de Paris X-Nanterre actuellement en délégation au CNRS (CERSA, université de Paris II, Panthéon-Assas), expert auprès du Conseil de l'Europe.

1 Cet article reprend et développe une partie de l'intervention faite au colloque « Droit de la ville et droit dans la ville », délégation interministérielle à la Ville Paris, le 29 mars 2001.

2 Pour une critique de la raison méritocratique voir : P. Bourdieu, « Le racisme de l'intelligence » in Questions de sociologie, Minuit, 1994.

3 Ce principe a été affirmé notamment par l'article 2 de la Déclaration universelle des droits de l'homme de 1948 et repris dans le Pacte international relatif aux droits civils et politiques de 1966 (articles 2 et 26). La Convention européenne des droits de l'homme le consacre dans son article 14.
} 
Les deux grandes doctrines politiques qui ont le plus influencé la modernité occidentale n'ont nullement contesté la primauté de l'égalité non seulement comme principe moral mais aussi en tant que facteur indispensable au développement personnel et à la cohésion sociale. Aussi bien le libéralisme que le marxisme ont combattu la société aristocratique fondée sur le privilège de la naissance, leur point de désaccord se situant dans une différence de pronostic plutôt que dans l'idéal d'égalité qui demeure une valeur commune. Alors que pour les libéraux le marché mettrait fin spontanément aux inégalités et aux injustices, pour les marxistes celui-ci ne fait qu'accentuer les différences entre les classes sociales. Nécessairement au service de la bourgeoisie, le marché serait par définition producteur d'inégalités. Dès lors, la tâche d'assurer la justice reviendrait au prolétariat, considéré par le marxisme comme seul moteur du changement et auteur exclusif de la révolution égalitariste.

La crise des systèmes d'interprétation politique globale a permis de poser la question d'une façon plus modeste. Aujourd'hui, il ne s'agit pas tant d'accomplir parfaitement l'idéal d'égalité matérielle, expérience menant souvent à des glissements de type autoritaire ${ }^{1}$, mais plutôt d'assurer les conditions d'une absence de discriminations dans l'accès aux droits, aux fonctions, aux pouvoirs et aux ressources culturelles, économiques et sociales.

Forme atténuée de l'égalité, la non-discrimination se contente de combattre les différentiations arbitraires par la création d'un climat général d'équité de chances. Puisqu'aucun système politique ne s'est montré capable d'assurer l'égalité des résultats, il semble nécessaire de se consacrer à la tâche menant de l'égalité formelle à l'égalité de possibilités. L'égalité des chances ${ }^{2}$ et la lutte contre les discriminations ${ }^{3}$ semblent donc compléter, plus ou moins efficacement, la notion traditionnelle d'égalité de droit.

\section{1: Exclusion, discrimination ou ségrégation: la question terminologique}

Avant d'analyser les questions proprement juridiques, un certain nombre de précisions terminologiques s'impose. D'une manière générale, les notions d'exclusion, de discrimination, de ségrégation, ou encore de marginalisation renvoient à des systèmes, des critères, des dispositions et des pratiques qui permettent, facilitent ou encouragent un traitement moins favorable envers des personnes se trouvant dans des situations semblables.

1 L'égalitarisme stalinien représente l'exemple extrême.

2 G. Koubi et G. Guglielmi (dir.), L'égalité des chances, La Découverte, 2000

3 Sur les rapports entre marché, vie privée et discriminations au travail voir la remarquable étude d'Olivier De Schutter, Discriminations et marché du travail. Liberté et égalité dans les rapports d'emploi, PIE - Peter Lang, Bruxelles, 2001. 
Du point de vue étymologique, le terme exclusion appartient à l'ensemble des mots dérivés de la racine latine «clau » (clef) exprimant l'idée de fermeture. Exclure signifie donc enfermer quelqu'un dehors, le mettre à l'extérieur en érigeant une barrière entre lui et les autres.

Le vocable discrimination trouve quant à lui sa racine dans le mot «crible » qui, dans sa version grecque, donne le terme krinein « séparer », «choisir », « décider » et en latin cribrum « le crible » ainsi que le verbe cernere « passer au crible ». Sous l'angle strictement étymologique, la discrimination est donc l'action de distinguer, de séparer. Si à l'origine le verbe discriminare n'a aucune connotation négative et renvoie tout simplement à une opération matérielle ou intellectuelle consistant à classer des objets en fonction d'un critère préétabli, dans sa version moderne il dénote «le fait de séparer un groupe social des autres en les traitant plus mal » ${ }^{1}$.

Enfin, le terme ségrégation provient de la famille latine grex, gregis « réunion d'animaux ou d'individus de même espèce $»$. Le verbe adgregare signifie «adjoindre à la troupe », congregare «réunir en une troupe » et segregare « séparer de la troupe ». La ségrégation est «l'action de mettre à part, le fait de séparer ». Le terme est habituellement utilisé pour faire référence à la séparation raciale (apartheid). "L'idée de ségrégation... s'appuie sur le slogan "Égaux mais différents". On sait que l'idée "d'égalité dans la différence" en fait manifeste toujours un refus de l'égalité. La ségrégation a amené aussitôt la discrimination » (S. de Beauvoir) ${ }^{2}$. "Discriminer, $c$ 'est distinguer, différencier, soit tenir pour distinct ou différent et, par conséquent, traiter distinctement ou différemment. L'opération de discrimination peut être mentale (faculté intellectuelle) ou matérielle (pratique concrète). La coupure est, par définition, moins abstraite dans la ségrégation : ségréguer, c'est étymologiquement, séparer du troupeau, instaurer une distance physique entre une partie (un ou plusieurs éléments) et le reste du groupe " ${ }^{3}$. La ségrégation représente donc la forme spatiale de la discrimination.

Tous ces termes véhiculent la même idée d'injustice et d'inégalité. Mais, alors que la notion d'exclusion fait référence à une mise à l'écart pour des raisons essentiellement écononiques (chômage, pauvreté, marginalité...), celle de discrimination renvoie à une dégradation de type juridique qui se manifeste par l'affaiblissement des droits subjectifs. Il est ainsi permis de considérer que l'exclusion constitue une atteinte à la valeur solidarité, la ségrégation à la valeur liberté (en particulier à la liberté de circulation) et la discrimination une mise en cause de la valeur égalité.

1 Source : Le Petit Robert.

2 Cité dans Dictionnaire Robert de la langue française, 1990.

3 De Rudder V., Poiret Ch., Vourc'h $\mathrm{F}$., L'inégalité raciste. L'universalité républicaine à l'épreuve, PUF, Paris, 2000, p. 49. 
Il existe un lien étroit entre ces concepts ; très fréquemment en effet, un phénomène d'exclusion est accompagné de discrimination et de ségrégation. Ainsi, une personne à la peau noire, surendettée et habitant dans une cité de banlieue est très souvent exclue et ségréguée et elle peut de surcroît être victime de discriminations. Néanmoins, résoudre le problème de l'exclusion n'implique pas nécessairement de mettre fin aux phénomènes discriminatoires. Dans le même ordre d'idées, une femme blanche d'origine européenne de classe moyenne par exemple, peut trouver plus de difficultés à accéder à un emploi qu'un homme dans la même situation. En outre, un gay ou une lesbienne appartenant à une élite économique et bénéficiant de tous les privilèges liés à leur condition sociale peuvent se trouver dans une situation de discrimination lorsqu'il ou elle décide de formaliser par le mariage l'union avec son compagnon ou sa compagne. Autrement dit, la disparition de l'exclusion ne constitue pas un gage d'affaiblissement de la discrimination. Les sociétés assurant aujourd'hui une plus grande égalité économique et sociale ne sont pas à l'abri de manifestations ou de pratiques discriminatoires. De même, une politique antidiscriminatoire efficace n'est pas en mesure de garantir la consolidation d'une égalité dans le marché.

Nous n'aborderons pas ici la problématique de l'exclusion ' ni celle relative à la ségrégation ; nous nous consacrerons exclusivement à l'analyse des discriminations, et ce, sous l'angle juridique.

\section{图 Le principe d'égalité et de non-discrimination}

Vivant dans un univers marqué par la diversité, nous chercherons en vain l'égalité dans les faits. Biologiquement, morphologiquement, économiquement, culturellement, sexuellement, la différence est en effet de mise. C'est en opposition à cette réalité factuelle, et en essayant de la dépasser, que le droit proclame cette fiction politique constituant l'un des fondements de la société démocratique : l'égalité. Suivant la métaphore de John Rawls, celle-ci consiste à jeter un voile d'ignorance sur l'ensemble des individus de telle sorte qu'ils soient traités, et qu'ils acceptent à leur tour de traiter les autres, sans aucune considération relevant de circonstances particulières ${ }^{2}$. Ce principe d'égalité formelle n'implique cependant pas le renoncement aux traitements différentiels ; parfois, la différence peut être légalement instituée. Ainsi, les mineurs ou les incapables bénéficient d'un traitement d'exception et sont placés sous tutelle afin de protéger leurs intérêts. De même, et pour lutter contre les inégalités matérielles, le droit autorise la mise en place des mesures correctrices qui peuvent constituer des inégalités : par exemple

\footnotetext{
1 Pour une analyse de cette question voir l'ouvrage collectif dirigé par S. Paugam, L'exclusion l'état des savoirs, La Découverte, Paris 1993.

2 A Theory of Justice, Harvard University Press, 1971.
} 
lorsque les individus bénéficient d'abattements fiscaux du seul fait de vivre dans des territoires éloignés de la métropole, quand certains emplois sont réservés aux jeunes ou bien encore lorsque des dispositions spéciales d'accès à la fonction publique sont mises en place pour les femmes ayant un ou plusieurs enfants.

Le Conseil constitutionnel a cependant encadré le principe de traitement différentiel : "Considérant que, si le principe d'égalité devant la loi ne fait pas obstacle à ce qu'une loi établisse des règles non identiques à l'égard des catégories de personnes se trouvant dans des situations différentes, il $n$ 'en est ainsi que lorsque cette non-identité est justifiée par la différence de situation » ${ }^{1}$. En principe donc, tous les individus doivent être traités de la même manière par la norme juridique (égalité dans la loi). La promotion d'un droit commun constitue la principale tâche du législateur. Le droit d'exception représentant une situation résiduelle, la dérogation au droit commun doit dès lors être maniée avec la plus grande prudence. Cela étant, un traitement différent ne constitue pas en lui-même une discrimination. La dimension discriminatoire n'apparaît en effet que si un tel traitement n'est pas légitimement justifié, autrement dit lorsqu'il est arbitraire. L'histoire du droit est en partie celle de l'évolution de la distinction (différentiation légitime) vers la discrimination (différentiation illégitime) de telle sorte qu'aussi bien les catégories que les domaines de différentiation justifiée ont été réduits, augmentant par-là même les cas de distinction arbitraire. Ainsi par exemple, alors que pendant des siècles le traitement différencié des esclaves apparaissait comme moralement et juridiquement justifié dans les sociétés colonialistes, la non-appartenance à la religion officielle dans ces mêmes sociétés ne pouvait pas être source d'un traitement juridique moins favorable. En effet, les discriminations fondées sur les croyances religieuses ont été l'une des premières distinctions considérées comme insupportables et arbitraires méritant une sanction juriđique. Ainsi, à l'apogée des empires coloniaux, les traités de Westphalie de 1648 contenaient des dispositions relatives à la liberté religieuse, mais il faudra attendre 1848 pour que la France décide d'abolir l'esclavage dans ses colonies. Après l'appartenance religieuse, la race est devenue à son tour une catégorisation formellement interdite par le droit interne et le droit international. Plus tard, seront également perçues comme illégitimes tout d'abord les différenciations fondées sur la nationalité (minorités nationales au sein d'un État), puis celles fondées sur le sexe.

Cette évolution se retrouve au sein du droit communautaire. En effet, dans le cadre de sa compétence économique et sociale, la Communauté européenne puis l'Union européenne ont construit un système juridique de protection contre les discriminations fondées sur la nationalité et sur le sexe afin d'assurer l'égalité de chances aux travailleurs européens, hommes et

1 Décision 87-232 CONS. CONST. 7 janvier 1988 RJC p. 319. 
femmes. En 1999, avec la ratification du traité d'Amsterdam par l'ensemble des États de l'Union, la protection s'est également élargie au handicap, à l'âge et à l'orientation sexuelle, de telle sorte qu'aujourd'hui, tout traitement différentiel des personnes en raison de leurs croyances, leurs origines ${ }^{1}$, leur race, leur sexe, leur âge, leur handicap ou leur orientation sexuelle est devenu un acte arbitraire, susceptible de constituer une discrimination. La seule exception au principe consiste à traiter d'une manière plus favorable des catégories historiquement discriminées. Ainsi, l'affirmative action (discrimination positive) résulte de la mise en place de mesures correctrices qui, par le biais d'une inégalité formelle, tendent à rendre efficace l'égalité matérielle.

L'évolution juridique ne porte pas seulement sur les catégories discriminées, elle concerne également les domaines dans lesquels les traitements différentiels sont prohibés. Si la liberté de conscience fut le premier des domaines protégés, de nos jours ce n'est pas uniquement la vie privée qui se trouve couverte par cette protection. En effet, le travail et l'emploi représentent actuellement les domaines au sein desquels les traitements distincts peuvent être considérés comme arbitraires et par conséquent constituer une discrimination. En outre, cette dernière pourra être formelle ou matérielle : elle est formelle lorsque la différentiation arbitraire se trouve dans le texte même de la règle et matérielle dès lors que l'arbitraire concerne les effets concrets de la norme. Dans le premier cas de figure (discrimination formelle), et pour déterminer si effectivement un traitement différentiel constitue une discrimination, il convient d'opérer un examen à trois dimensions : tout d'abord, il est nécessaire de considérer le groupe ou les personnes distinguées afin d'apprécier si ceux-ci se trouvent dans une situation objectivement différente, ensuite, il faut s'arrêter sur le domaine, objet de différentiation ; enfin doivent être analysées les raisons qui justifient un traitement exceptionnel ${ }^{2}$.

Les organismes chargés de contrôler la conformité d'une règle au principe d'égalité varient selon les pays. Aux États-Unis cette tâche incombe à la Cour suprême, en France c'est au Conseil constitutionnel et au Conseil d'État. Au niveau européen, ce sont la Cour européenne des droits de l'homme et la Cour de justice des communautés européennes qui s'occupent de la mise en œuvre effective du principe d'égalité et de non-discrimination. En outre, chaque organe judiciaire a ses propres critères pour mesurer la qualité des normes aux trois niveaux indiqués plus haut. Ainsi, en tenant compte du bloc de constitutionnalité (Déclaration de 1789,

1 "Loin d'être redondante par rapport à l'interdiction de discrimination à raison de la race, la référence à l'origine ethnique dans l'article $13 \mathrm{CE}$ désigne - selon l'inspiration que la House of Lords britannique est allée puiser, en 1983 dans la jurisprudence néo-zélandaise à propos de cette notion - l'appartenance de l'individu à un groupe que caractérisent une histoire et un ensemble de traditions communes, que les membres du groupe ont souhaité maintenir vivants ", O. De Schutter, op. cit., p. 46

2 D. Lochak, "Réflexions sur la notion de discrimination ", Droit Social $\mathrm{n}^{\circ} 11$, novembre 1987. 
Préambule de la Constitution de 1946 et Constitution de 1958), les juridictions françaises estiment que toute différence de traitement fondée sur la race, la religion, les croyances ou le sexe doit être automatiquement considérée comme discriminatoire (sauf évidement les mesures de « discrimination positive »). La Cour suprême des États-Unis procède quant à elle au contrôle par le biais de la notion de « catégorie suspecte ». Lorsqu' une différence de traitement opère au détriment d'un groupe envisagé comme historiquement défavorisé, celle-ci est considérée comme suspecte et dès lors soumise à un contrôle particulièrement strict. Aux États-Unis sont considérées comme suspectes les distinctions fondées sur la race, l'origine nationale ou la qualité d'étranger. En revanche restent en dehors du contrôle renforcé des catégories telles que, le sexe, le handicap et l'orientation sexuelle, cela signifie que les femmes, les handicapés et les homosexuels se trouvent moins bien protégés par la plus haute juridiction étatsunienne. Enfin au niveau européen, c'est par l'application de l'article 14 de la Convention européenne des droits de l'homme que la Cour de Strasbourg procède au contrôle du principe de non-discrimination. Cet article dispose que "La jouissance des droits et libertés reconnus dans la présente Convention doit être assurée, sans distinction aucune, fondée notamment sur le sexe, la race, la couleur, la langue, la religion, les opinions politiques ou toutes autres opinions, l'origine nationale ou sociale, l'appartenance à une minorité nationale, la fortune, la naissance ou toute autre situation ». Enfin, la Cour de justice des Communautés européennes a traditionnellement agi en faveur des deux groupes protégés par les traités constitutifs de l'Union : les femmes et les ressortissants des États membres vivant dans d'autres pays de l'Union. Comme nous l'avons souligné, ce n'est que très récemment (1999) que l'Union européenne a élargi sa compétence antidiscriminatoire à d'autres domaines que le sexe et la nationalité, et ce, au-delà des implications purement économiques ou sociales.

\section{La compétence communautaire en matière de politique antidiscriminatoire}

Si le droit communautaire accorde au principe d'égalité une place centrale et si le traité de Rome fait de l'interdiction des discriminations fondées sur la nationalité et sur le sexe la base d'un combat contre l'inégalité, il s'agit avant tout d'une préoccupation de type économique ayant comme objectif le bon fonctionnement du Marché commun ${ }^{1}$. Ce n'est qu'à l'occasion du mandat explicite du traité de Maastricht qu'une Conférence intergouvernementale s'est ouverte le 29 mars 1996 à Turin, pour la première fois la

1 A. Mattera, «La libre circulation des travailleurs à l'intérieur de la Communauté euro péenne ", Revue du Marché unique européen $n^{\circ} 4,1993$ 
question de la lutte contre les discriminations y a été évoquée d'une façon générale et sous un angle éminemment politique. À la suite de cette Conférence, il a été convenu de réunir un Conseil européen. Celui-ci a permis la conclusion du traité d'Amsterdam (entré en vigueur le $1^{\text {er }}$ mai 1999 après la ratification des pays membres), qui énonce dans son article 13 une clause antidiscriminatoire générale : "Sans préjudice des autres dispositions du présent traité et dans les limites des compétences que celui-ci confère à la Communauté, le Conseil, statuant à l'unanimité sur proposition de la Commission et après consultation du Parlement européen, peut prendre les mesures nécessaires en vue de combattre toute discrimination fondée sur le sexe, la race ou l'origine ethnique, la religion ou les convictions, un handicap, l'âge ou l'orientation sexuelle ». Ce dispositif complète la Charte communautaire des droits sociaux fondamentaux des travailleurs ${ }^{1}$ en donnant aux politiques antidiscriminatoires une assise juridique plus concrète.

La vision classique qui consistait jusqu'ici à considérer que la Convention européenne de droits de l'homme s'occupait des droits fondamentaux au sein de la grande Europe (Conseil de l'Europe), tandis que les traités constitutifs de l'Union se limitaient à une tâche plutôt économique a été abandonnée au profit d'une conception du droit communautaire lui aussi consacré aux libertés et aux droits fondamentaux. Bien que reconnus depuis longtemps par la Cour de justice des Communautés européennes, ce n'est qu'avec l'entrée en vigueur du traité d'Amsterdam puis de la Charte européenne des droits fondamentaux ${ }^{2}$, que ceux-ci feront incontestablement partie intégrante de l'ordre juridique de l'Union ${ }^{3}$.

Cependant, il est nécessaire de nuancer la portée de l'article 13. En effet, celui-ci n'est pas d'application directe, il ne peut pas être invoqué devant un juge national comme c'est le cas pour l'article 141 concernant l'égalité de rémunération entre hommes et femmes ou pour l'article 12 relatif aux discriminations fondées sur la nationalité. Les mesures découlant de l'article 13 doivent être prises à l'unanimité du Conseil des ministres ce qui implique une recherche de consensus parmi les quinze États membres. Cette politique fondée sur le consensus semble expliquer la portée limitée des directives adoptées ultérieurement. Par ailleurs, la phrase avec laquelle commence l'article 13, ( Sans préjudice des autres dispositions du présent traité ») fait référence aux dispositions spécifiques relatives aux discriminations fondées

1 Le préambule de la Charte de 1989 établit: « considérant que, pour assurer l'égalité de traitement, il convient de lutter contre les discriminations sous toutes leurs formes, notamment celles fondées sur le s'exe, la couleur, la race, les opinions et les croyances, et que, dans un esprit de solidarité, il importe de lutter contre l'exclusion sociale".

2 L'article 21 établit: "Est interdite toute discrimination fondée notamment sur le sexe, la race, la conleur, les origines ethniques ou sociales, les caractéristiques génétiques, la langue, la religion ou les' convictions, les opinions politiques ou toute autre opinion, l'appartenance à une minorité nationale, la fortune, la naissance, un handicap, l'âge ou l'orientation sexiclle...".

3 Pour une analyse plus approfondie voir: Mark Bell, "Equality and Diversity: Anti-discrimination Law after Amsterdam", in J. Shadow, Social Law and Policy in an Evoluing European Union, Hart, 2000. 
sur le sexe ${ }^{1}$ et sur la nationalité ${ }^{2}$; des catégories bien plus anciennes qui bénéficient déjà d'un arsenal juridique protecteur conséquent. Les deux directives qui mettent en place le mandat de l'article 13 ne couvrent donc ni les discriminations envers les femmes ni celles et à l'égard d'autres ressortissants de l'Union.

\section{Les discriminations sanctionnées dans l'ensemble des domaines de compétence communautaire mais pour des catégories limitées (approche verticale)}

Elles ne concernent que les discriminations liées à la « race » et aux origines « origines ethniques ».

La première directive ${ }^{3}$ portant sur la « mise en æuvre du principe de l'égalité de traitement entre les personnes sans distinction de race ou d'origine ethnique » trouve sa source non seulement dans le principe de l'article 13 mais également dans l'invitation faite à la Commission lors du Conseil européen de Tamperer en 1999, de présenter un programme juridique de lutte contre le racisme et la xénophobie. Malgré la dimension politique insufflée par le traité d'Amsterdam, c'est tout d'abord pour des questions matérielles que la Commission a édicté cette directive. En effet, les premières valeurs rappelées dans l'exposé des motifs sont « l'emploi » et la « protection sociale », ce n'est que par la suite que sont invoquées la « qualité de vie » et la « cohésion économique et sociale » et enfin plus loin « la solidarité ». L'approche communautaire semble, en ce sens, compléter celle de la Cour européenne des droits de l'homme qui pose la question du principe d'égalité et non-discrimination en tant que tel.

La protection mise en place par la directive va au-delà de l'accès aux activités salariées, ellè s'étend également aux activités non-salariées et à des domaines tels que l'éducation, la protection sociale, la santé, les avantages sociaux, la fourniture de biens et services et l'accès à ces demiers. Elle est destinée non seulement aux ressortissants européens mais plus généralement à toutes les personnes habitant sur le sol de l'Union. Elle s'applique aussi bien aux personnes physiques qu'aux personnes morales, elle est valable tout autant pour le secteur public que privé.

Les discriminations sanctionnées par la directive peuvent être directes ou indirectes. Une discrimination directe se produit « lorsque, pour des raisons

1 Articles 141 et 137 (1).

2 Article 12.

3 Directive 2000/43/CE du Conseil du 29 juin 2000, JOCE nº L. 180 du 19 juillet 2000. 
de race ou d'origine ethnique, une personne est traitée de manière moins favorable qu'une autre ne l'est, ne l'a été ou ne le serait dans une situation semblable». Une discrimination est considérée comme indirecte "lorsqu'une disposition, un critère ou une pratique apparemment neutre est susceptible d'entraîner un désavantage particulier $»$. Un exemple de discrimination indirecte a été donné par la Cour de justice des Communautés européennes dans l'arrêt Bilka-Kaufhaus du 13 mai 1986. En l'espèce, la discrimination résultait du fait que les travailleurs à temps partiel ne bénéficiaient pas des mêmes avantages que les travailleurs à temps plein et que majoritairement les femmes occupaient des postes à temps partiel, ces dernières pouvaient donc se considérer comme victimes d'un traitement discriminatoire ${ }^{1}$. Mais, à la différence de cette jurisprudence et de la directive $97 / 80$ qui, en matière de discrimination fondée sur le sexe ${ }^{2}$, consacre la méthode statistique ${ }^{3}$, les nouvelles directives, bien que recherchant le même résultat, n'utilisent guère la même approche. Il ne s'agit plus de condamner l'impact disproportionné mesuré en fonction de critères quantitatifs (nombre de femmes notamment) mais de le faire en tenant compte du désavantage particulier qu'une mesure apparemment neutre est susceptible de provoquer dans l'une des catégories visées par l'article 13.

La directive va encore plus loin en considérant que constituent des discriminations non seulement les actions directes et les effets indirects, mais également le harcèlement défini comme un " comportement indésirable [...] qui a pour objet ou pour effet de porter atteinte à la dignité d'une personne et de créer un environnement intimidant, hostile, dégradant, humiliant ou offensant ».

La norme européenne autorise néanmoins les États à faire une exception à son application lorsqu' une différence de traitement se justifie pour des raisons liées à la nature d'une activité professionnelle pour autant que l'objectif soit légitime et que l'exigence soit proportionnée. Elle permet aussi la mise en œuvre des discriminations positives (appelées «actions positives ») destinées à prévenir ou à compenser des désavantages liés à la race ou à l'origine ethnique. À la différence de celles relatives à la discrimination indirecte, les mesures d'action positive ne sont pas obligatoires, la Commission laisse leur mise en place à la libre appréciation des États.

1 La Cour s'exprime de la sorte : "L'article 119 est violé lorsqu'tue mesure frappe un nombre beaucoup plus élevé de femmes que d'hommes, à moins que l'entreprise n'établisse que la mesure s'explique par des facteurs objectivement justifiés et étrangers à toute discrimination fondée sur le sexe ».

2 Cette directive définit la discrimination indirecte comme suit: « Une discrimination indirecte existe lorsqu'une disposition, un critere ou une pratique apparemment neutre affecte une proportion nettentent plus élevée de personnes d'un sexe, à moins que cette disposition, ce critère ou cette pratique ne soit appropriée et nécessaire et ne puisse être justifiée par des facteurs objectifs indépendants du sexe des intéressés" ".

Voir M.-T. Lanquetin, "La preuve de la discrimination : l'apport du droit communautaire », Droit Social, 1995.

3 Méthode utilisée par la CJCE depuis 198 I s'inspirant d'une décision de la Cour suprême des Etats-Unis: Griggs v. Duke Power Co. 1971. 
Enfin, deux dispositions méritent une attention particulière ; tout d'abord celle qui concerne la charge de la preuve devant la juridiction civile : la directive pose en effet le principe qu'une fois établis « des faits qui permettent de présumer l'existence d'une discrimination directe ou indirecte, il incombera à la partie défenderesse de prouver qu'il n'y a pas eu violation du principe de l'égalité de traitement ». Ensuite, la directive oblige les États membres à édicter des mesures contre les rétorsions afin d'éviter des représailles de la part des employeurs. Avec la possibilité pour les associations ou les syndicats de se porter partie civile, la Commission entend aussi faciliter la protection des individus victimes de racisme.

\section{瞷 Les discriminations sanctionnées exclusivement dans le domaine de l'emploi mais pour l'ensemble des catégories (approche horizontale)}

La seconde directive ${ }^{1}$ "portant création d'un cadre général en faveur de l'égalité en matière d'emploi et de travail " fait référence aux discriminations fondées sur la religion ou les convictions, un handicap, l'âge ou l'orientation sexuelle. L'exposé des motifs rappelle que le principe d'égalité entre hommes et femmes ne fait pas partie de la directive, lequel principe est prévu par plusieurs autres dispositions communautaires. La directive spécifie également qu'elle ne porte pas atteinte aux dispositions nationales fixant les âges de la retraite ainsi qu'à celles relatives à l'aptitude physique requise par l'armée. De même, la norme européenne ne vise pas les différences de traitement fondées sur la nationalité ou sur les conditions d'admission des ressortissants de pays tiers. Concernant les établissements religieux, la règle communautaire autorise une exception à son application afin de permettre notamment la sélection des candidats en fonction de l'appartenance religieuse sans que cela ne constitue une discrimination. Certaines mesures concernant l'âge peuvent également rester en dehors du domaine d'action de la directive. Une situation spécifique est prévue pour l'Irlande du Nord où, compte tenu de la spécificité des communautés religieuses, la norme communautaire n'est pas applicable à la loi nationale.

Par ailleurs, cette directive reprend les mêmes notions de discrimination directe ou indirecte ainsi que les prohibitions relatives au harcèlement et à l'incitation à la discrimination. Comme pour la directive précédente, elle permet l'adoption de mesures destinées à prévenir ou à compenser des désavantages de l'une des catégories protégées (discrimination positive). Concernant la procédure, la charge de la preuve ainsi que les mesures

1 Directive 2000/78/CE du Conseil du 27 novembre 2000, JOCE n ${ }^{\circ}$ L. 303 du 2 décembre 2000 
contre les rétorsions, cette directive reprend les mêmes dispositifs que ceux de la précédente.

D'une façon générale, l'apport des directives est particulièrement significatif sur quatre points fondamentaux. :

- tout d'abord, elles introduisent la notion de discrimination indirecte permettant ainsi de sortir de la logique subjective selon laquelle il serait nécessaire de prouver une volonté discriminatoire. Ce sont désormais les effets discriminatoires et non pas les causes qui comptent au moment de déterminer la qualité d'un fait ou d'une norme ;

- en outre, les directives instaurent le renversement de la charge de la preuve, en matière civile, libérant la victime de discriminations d'un tel devoir ;

- les directives permettent également aux associations et aux syndicats de se porter partie civile à la place de la victime ;

- et enfin elles prévoient des mesures contre les éventuelles vengeances des employeurs.

En favorisant le dialogue social, la mise en place des mesures correctrices et la création d'instances médiatrices, les directives mettent à la portée des citoyens d'importantes ressources juridiques les protégeant contre les éventuels agissements discriminatoires.

Enfin, l'action européenne en matière de lutte contre les discriminations est complétée par un programme d'action sur six ans (2001-2006) ayant comme objectif notamment d'encourager les mesures de prévention, de faire mieux comprendre les questions liées à la discrimination, de favoriser l'échange d'information et la mise en réseau. Dans ce domaine, la répression n'a de sens qu'avec la mise en place d'une véritable politique de prévention.

\section{诵 La situation en France}

Les directives communautaires viennent compléter le paysage juridique français qui, depuis 1972, n'a cessé de mettre en accusation les comportements discriminatoires.

La loi no 72-546 du $1^{\text {er }}$ juillet 1972, outre le fait de considérer comme injurieux et diffamatoire tout propos raciste, punit les discriminations consistant en un refus d'accès à un droit ou à un service, motivé par des considérations raciales, nationales ou religieuses. Une loi du 11 juillet 1975 a élargi la répression des discriminations à celles fondées sur le sexe et la situation familiale. Deux ans plus tard, le 7 juin 1977, une nouvelle loi a introduit dans l'ancien Code pénal l'incrimination d'entrave à une activité économique pour les motifs mentionnés plus haut. Par la suite, la loi $\mathrm{n}^{\mathrm{o}}$ 85-772 du 25 juillet 1985 punira également les discriminations fondées sur les mœurs puis, un texte du 13 janvier 1989 (complété par la loi 
$\mathrm{n}^{\circ}$ 90-602 du 12 juillet 1990), sanctionnera celles fondées sur le handicap. En 1992, une nouvelle loi a complété le dispositif protecteur dans le droit du travail (article L. 122-45).

Les articles 225-1 à 225-4 et 432-7 du nouveau Code pénal ${ }^{1}$ ont simplifié la présentation des atteintes à la personne en créant un cadre général à caractère transversal. Désormais, constitue en effet une discrimination toute distinction opérée entre les personnes physiques ou morales en raison de leur origine, sexe, situation de famille, état de santé, mœurs, opinion politique, activité syndicale, appartenance vraie ou supposée à une ethnie, une race ou une religion déterninée. L'âge, le patronyme, l'apparence physique et l'orientation sexuelle n'étaient pas expressément mentionnés par la loi française. Un système de preuve, trop contraignant pour le plaignant, empêchait de plus la sanction efficace des comportements discriminatoires ${ }^{2}$.

L'ouverture du débat sur l'amélioration des dispositifs juridiques de lutte contre les discriminations constitue l'un des objectifs affichés par le gouvernement socialiste. Avant même la parution des directives communautaires dans la matière, les autorités françaises s'étaient engagées dans un processus de reforme de la loi permettant d'accroitre les garanties contre les discriminations. L'ancienne ministre chargée des Affaires sociales, Martine Aubry, avait tout d'abord proposé une loi générale sur la modernisation sociale au sein de laquelle ont été introduits des amendements aux dispositifs antidiscriminatoires existants. Finalement, le gouvernement a opté pour faire sortir les articles relatifs aux discriminations du projet de loi sur la modernisation sociale en confiant au Parlement le soin d'élaborer un texte spécifique consacré exclusivement à « la lutte contre les discriminations ${ }^{3}$. Cette proposition de loi a ainsi repris les articles 49,50 et $51 \mathrm{du}$ projet de loi Aubry ${ }^{4}$, en laissant toutefois dans ce dernier les mesures relatives aux discriminations dans la location des logements ainsi que celles concernant le harcèlement moral ${ }^{5}$.

De son côté, la justice n'a pas attendu la reforme pour interpréter la loi française selon les critères proposés par le droit communautaire. En effet, depuis un arrêt du 28 novembre 1999, la chambre sociale de la Cour de cassation applique régulièrement le système de preuve européen. Ce changement législatif et jurisprudentiel constitue une révolution dans la culture de la preuve des discriminations. Jusqu'alors, en effet, le système de preuve reposait au

1 Loi 2001-1066 du 16 novernbre 2001, JO du 17 novembre 2001.

2 La situation au pénal demeure la même malgré la réforme de la loi

3 Proposition de loi relative à la lutte contre les discriminations $n^{\circ} 2566$, déposée le 13 septembre 2000 par Jean Le Garrec. Après deux lectures aux assemblées, l'échec de la commission mixte paritaire et une nouvelle lecture aux assemblées, la proposition est finalement adoptée devenant ainsi la loi $\mathrm{n}^{\circ} 2001-1066$ du 16 novembre 2001 relative à la lutte contre les discriminations ( $J O \mathrm{n}^{\circ} 267 \mathrm{du} 17$ novembre 2001 , p. 18311).

4 Devenu loi de modernisation sociale, 21 décembre 2001.

5 La loi de modernisation sociale modifie les lois du 23 décembre 1986 et du 6 juillet 1989 (Ch. III art. 158 lutte contre les discriminations dans la location des logements). Le chapitre IV de cette loi est consacré à la lutte contre le harcèlement moral au travail. 
niveau pénal sur l'intentionnalité et, même dans le domaine civil, il incombait au demandeur de produire la preuve de la discrimination. Cette situation rendait la loi française pratiquement impuissante. Non seulement ce n'est qu'exceptionnellement qu'une telle affaire était soumise aux tribunaux, mais qui plus est, elle se traduisait par un échec pour la victime.

La loi du 16 novembre 2001 relative à la lutte contre les discriminations essaye de pallier ces lacunes. Composée de onze articles, elle modifie les dispositions relatives aux discriminations du Code de travail, du Code pénal, du Code de la sécurité social et de la loi du 13 juillet 1983 sur le statut des fonctionnaires !. Outre la consécration des «catégories» telles que l'apparence physique, le patronyme, l'orientation sexuelle et l'âge, la nouvelle loi autorise les associations syndicales à exercer en justice toutes les actions qui naissent de l'article L. 122-45 du Code de travail, c'est-à-dire tout ce qui est relatif aux discriminations. Les associations de lutte contre les discriminations peuvent également exercer l'action «sous réserve qu'elles justifient d'accord écrit de l'intéressé ». Si l'action en justice aboutit, le licenciement sera considéré comme nul et la réintégration de plein droit du salarié sera immédiate. En outre, les salariés qui ne souhaitent pas réintégrer leur emploi, bénéficient d'une indemnité qui ne peut pas être inférieure aux salaires de six derniers mois plus les indemnités correspondant au licenciement s'ils comptent deux ans d'ancienneté ininterrompue au service du même employeur.

Cependant, la loi ne prévoit pas la création d'une autorité compétente en matière de discrimination, laissant ainsi aux juges le contrôle exclusif des faits (en revanche, un service d'accueil téléphonique gratuit contre les discriminations raciales est institutionnalisé par la $1 \mathrm{loi}^{2}$ ). Elle introduit la notion de discrimination indirecte sans pour autant la définir ${ }^{3}$, ce qui permet de penser que les juges se référeront aux directives communautaires. Il est toutefois permis de regretter la rédaction confuse du texte qui semble exiger du salarié la présentation des faits laissant supposer l'existence d'une discrimination indirecte. Cette situation empêcherait le juge français

1 Ne modifiant pas le Code de la procédure pénale, les nouvelles catégories n'y sont pas introduites. Cette situation ne devrait pas empêcher les associations de défense de droits des homosexuels puisque le code mentionne la possibilité de se porter partie civile (sous la dénomination de mours) dans les infractions pénales commises contre les personnes supposées homosexuelles. Mais pour les autres catégories telles l'apparence physique ou l'âge rien n'est prévu.

2 Celte ligne existe déjà depuis quelques mois $\left(\mathrm{n}^{0} 114\right)$, elle est gérée par le Groupe de recherche et de lutte contre les discriminations.

3 Il semblerait que le législateur n'ait pas voulu tenir compte de la définition donnée par les directives puisque cela impliquerait de se référer à des catégories de la population (situation interdite par le Constitution française). Pour les mêmes raisons « universalistes " rien non plus n'est prévu sur la discrimination positive (encouragée par les directives). L'unité de la République et la culture juridique française empêcheraient l'institutionnalisation de ce type de mesure. Cette idée très tépandue nous semble mériter bien de nuances. En effet, d'une part de telles règles existent déjà en droit français (parité, emplois jeune, quotas pour les handicapés, privilèges pour les fonctionnaires mères...) et d'autre part comme le signale M.-T. Lanquetin, (cf. article précité), il ne s'agit pas de construire des catégories qui enfermeraient a priori des individus en fonction de certains traits mais de constater a posteriori (et dans le cadre du contentieux) la récurrence d'une discrimination dans certains groupes. 
de condamner comme discriminatoire une mesure « apparemment neutre » indépendamment du rapport aux faits. Autrement dit, le souhait exprimé par M.-T. Lanquetin ${ }^{1}$ lorsqu'elle considérait que les nouvelles directives permettraient « de ne pas attendre le constat de l'effet d'une mesure, mais peut-être de prendre conscience des risques d'une mesure et de lutter ainsi contre les préjugés» ne semble pas trouver une concrétisation dans la loi française.

Mal gré la recommandation explicite des directives, la loi française ne prévoit pas des mesures spécifiques « destinées à prévenir ou à compenser des désavantages » visés par la norme européenne ${ }^{2}$. Enfin, il est important de soul igner que les notions de discriminations indirectes et l'inversion de la charge de la preuve ne sont pas introduites dans le Code pénal.

\section{凬 Conclusion : la hiérarchie du mal}

En énonçant différentes catégories susceptibles de protection, l'article 13 du traité d'Amsterdam entend garantir la sauvegarde de chacune d'elles avec la même vigueur. L'approche horizontale semble avoir conduit la rédaction de cet article. Une telle approche consiste à considérer que l'ensemble de ces catégories (sexe, race, nationalité, orientation sexuelle, handicap...) doit être protégé d'une manière équivalente dans tous les domaines (emploi, services, éducation, logement...). Or, aussi bien dans les directives qu'au sein de la loi nationale, la protection n'est pas la même s'agissant des personnes appartenant, ou censées appartenir, à l'une ou l'autre des catégories. En effet, le combat contre les discriminations fondées sur le sexe dispose d'instruments juridiques vieux de plus de cinquante ans et les discriminations basées sur la nationalité ont été au cœur des préoccupations communautaires depuis le début de la création du Marché commun. De même, la lutte contre le racisme dispose d'un arsenal répressif et préventif sans précédent. Le mandat donné par le traité d'Amsterdam aurait permis de mettre au même niveau l'ensemble des discriminations et de concevoir une véritable politique antidiscriminatoire d'ensemble.

Or, à la lecture des directives communautaires, on aperçoit clairement la hiérarchie consacrée par la norme. En effet, aux catégories «sexe» et « nationalité » (déjà existantes et protégées par d'autres lois) viennent s'ajouter celles de «race» et d'《 origine ethnique », catégories qui

\footnotetext{
1 Cf. article précité.

2 Malgré la recommandation générale faite par les directives, il est toutefois important de souligner que seules les discriminations positives relatives au sexe trouvent une consécration formelle au sein de la Charte des droits fondamentaux de l'Union européenne. En effel, l'article 23 établit: "L'égalité entre les hommes et les femmes doit être assurée dans tous les domaines y compris en matière d'emploí, de travail et de rénunération. Le principe d'égalité n'empêche pas le maintien ou l'adoption de mesures prévoyant des avantages spécifiques en faveur du sexe sous-représenté ».
} 
méritent la plus haute protection communautaire. En ce qui concerne les domaines de protection, l'emploi garde également sa place privilégiée car il demeure le seul champ à se trouver couvert pour l'ensemble des catégories. Être un employeur sexiste, raciste ou xénophobe devient le comportement le plus durement sanctionné par la norme européenne. En revanche, être un enseignant homophobe ou un fournisseur antisémite laisse cette même norme indifférente. La création d'organismes de promotion de l'égalité de traitement est obligatoire uniquement pour les discriminations fondées sur la race et les origines ethniques; cette hiérarchie apparaît même dans les délais de mise en œuvre des directives. En effet, alors que pour la directive concernant les discriminations raciales, la commission oblige les États à la rendre effective au plus tard le 19 juillet 2003, pour celle relative aux autres catégories, les mêmes États peuvent le faire jusqu'au 3 décembre 2006. Cette situation n'est pas en conformité avec le mandat de l'article $13 \mathrm{du}$ traité d'Amsterdam. Encore une fois, le consensus politique européen semble se construire au prix d'un affaiblissement du principe d'égalité.

Bien que plus satisfaisante au niveau de l'énoncé de la loi, la situation française demeure néanmoins précaire. En effet, au-delà de l'action répressive prévue par les textes juridiques, la dimension préventive est toujours élaborée en fonction d'un système hiérarchique. Outre les mesures de discrimination positive à l'égard des femmes dans la représentation politique (parité) et des handicapés (quota obligatoire pour les entreprises), de nombreuses actions ont été mises en place pour lutter contre les discriminations raciales. Les institutions publiques consacrées à la lutte contre les discriminations telles le Haut Conseil de l'intégration, les commissions départementales d'accès à la citoyenneté (Codac) ou encore le tout récent groupement d'intérêt public (le Groupe d'étude et de lutte contre les discriminations, GELD), sont exclusivement chargées, malgré le caractère général de leurs dénominations, des discriminations raciales et ethniques. Les autres groupes discriminés demeurent dans une situation moins protégée ${ }^{1}$.

L'expérience internationale montre que les États qui ont à l'heure actuelle réussi à mettre en place des moyens efficaces (commissions, médiateurs, Ombudsman...) permettant de prendre conscience et d'affaiblir toutes les situations susceptibles de produire des discriminations, sont ceux qui ont le plus investi dans les actions extra-judiciaires capables de coordonner les politiques publiques aussi bien au niveau de l'accès à la justice, de la pédagogie et la coopération avec la société civile. De par leur plus grande souplesse, ces autorités parviennent à des résultats encourageants à travers la mise en place de programmes d'accès à l'égalité, de campagnes de prévention, d'enquêtes, d'assistance aux victimes, de la préparation des poursuites

1 La même situation est constatée s'agissant du discours de haine. En effet, alors que les propos racistes et antisémites sont condamnés par la loi en tant qu'injurieux ou diffamatoires, ceux à caractère sexiste ou homophobe se trouvent dépourvus de sanction juridique. 
judiciaires, de médiations auprès des entreprises ou encore de formations des policiers, magistrats, travailleurs sociaux...

Malheureusement en France, les propositions allant dans ce sens ' n'ont pas été retenues. Or, la répression des discriminations doit nécessairement être accompagnée d'une prévention, d'une sensibilisation, d'une écoute des victimes et d'une médiation en l'absence desquelles toute politique publique est vouée à l'échec.

1 J.-M. Belorgey, Lutter contre les discriminations, rapport à la ministre de l'Emploi et de la Solidarité, mars 1999. 